\title{
Permanent Magnet Machine Design Trade-offs to Achieve Sensorless Control at High Load
}

\section{Structured Abstract:}

Purpose - The aim of this paper is to introduce a new design optimization technique for a surface mounted permanent magnet (SMPM) machine to increase sensorless performance at high loadings by compromising with torque capability.

Design/methodology/approach - An SMPM parametric machine model was created and analysed by finite element analysis (FEA) software by means of the Matlab environment. Eight geometric parameters of the machine were optimized using genetic algorithms (GAs). The outer volume of the machine, namely copper loss per volume, was kept constant. In order to prevent sensorless performance loss at high loading, an optimization process was realized using two loading stages: maximum torque with minimum ripple at nominal load and maximum self-sensing capability at twice load. In order to show the effectiveness of the proposed technique, the obtained results were compared with the classical one-stage optimization realized for each loading condition separately.

Findings - With the proposed technique, fairly good performance results of the optimisation were obtained when compared with the one stage optimisations. Using the proposed technique, sensorless performance of the motor was highly increased by compromising torque capability for high loading. Additionally, this paper shows that the self sensing properties of a SMPM machine should be considered at the design stage of the machine.

Originality/value -In related literature, design optimization studies for the sensorless capability of SMPM motor are very few. By increasing optimisation performance, new proposed technique provides to achieve good result at high load for sensorless performance compromising torque capability.

Keywords:

sensorless control, design optimization, genetic algorithms, permanent magnet machine

\section{INTRODUCTION}

Due to the lack of motion sensors, sensorless control of AC motors is desirable in many applications in terms of cost and reliability. In order to realize sensorless control, the rotor position must be estimated using model based or saliency based methods. Model based methods rely directly or indirectly on the back-EMF induced by the rotor magnets in the stator windings of the machine. In low speed applications, due to the decreasing magnitude of the backEMF, sensorless performance becomes weak. Saliencybased signal injection methods have been proposed for low/zero speed applications (Lorenz, 2006; Ha et al., 2002; Yang and Lorenz, 2011; Fernandes et. al., 2010; Al-Nabi et al., 2013; Zhu and Gong, 2011; Wu et al., 2007). In this method, hf voltage is injected and the hf currents are modulated by the machine's magentic saliency from which the rotor position can be derived. However, the robustness (ie. no loss of saliency) and the quality of this position signal depends on the machine geometry and this has prevented industrial application. This paper considers a GA design procedure that allows position robustness and quality to be maintained. The procedure may compromise on torque density and this is part of the investigation. Robust, quality position "self-sensing" allows industry to manufacture and market PMSM drives having a robust, good zero-speed, fulltorque capability. Without such a machine design process, full-torque, zero-speed PM drives would not see application. This would prevent many applications (e.g. industrial servos, automotive motors, aerospace actuators) from benefiting from sensorless control. The rotor position of an electrical machine can be estimated if there is a form of magnetic saliency, dependent on the rotor position, which modulates the impedance seen from the machine power terminals. This saliency can either be in the rotor itself, due to the rotor geometry, or as a result of iron saturation in the rotor or stator due to the synchronously rotating magnetic flux. This saliency can be tracked by processing the current response to a test voltage signal injection overlaid on the main PWM excitation (Caner et al., 2011, 2013).

Within this work, a 12-slot-10-pole surface mounted permanent magnet (SMPM) synchronous machine was considered. Machines with sub-unity numbers of slots per pole per phase are very popular in industrial servo drives and automotive traction machines, as well as in high performance, fault-tolerant aerospace machines and other demanding applications. Some key advantages of such machines include short end-winding lengths, thus resulting in low copper utilization, a high copper packing factor, and low cogging torque, and can be designed to be fault-tolerant. The slot-pole combination considered in this work is a commonly used combination due to its relatively high winding factor and small pu cogging torque. Fractional slot SMPM machines, in general, have their phase inductance dominated by a stator slot leakage. This leads to the main dominant saliency component being due to stator iron saturation rather than due to the rotor's geometric features. A key characteristic of such fractional slot machines is their harmonic rich armature field. This leads to asynchronous rotating fields, which may induce significant rotor losses if not adequately designed. In addition to this, the stator magnetic material can become heavily saturated at high loading levels due to the relatively high slot leakage inductance.

Although interior permanent magnet (IPM) and synchronous reluctance motors (Guglielmi et al., 2006) are inherently salient, SMPM motors have little magnetic saliency due to the cylindrical rotor topology and the fact the magnets have a relative permeability of near that of air. The only saliency is that caused by the stator flux saturation in the vicinity of the permanent magnet. The saturation, the source of the little saliency, occurs at the stator core around the q-axis winding (Jang et al., 2003). Moreover, saliency can be easily degraded according to the loading conditions (Jang etal., 2001). Saliencies, resulting from the difference in incremental inductances in the direct and quadrature axes, are the main factors that influence sensorless performance 
for techniques based on high frequency injection. In SMPM machines, these are mainly a function of the magnetic material's non-linearity as the machines have an inherently negligible geometric saliency. A major issue with controlling the machines at high load arises due to the fact that this differential saliency tends to decrease with increased machine loading (Arellano-Padilla et al., 2010). Apart from ensuring that this saliency does not disappear within the loading range of the machine, it is also desirable to maximize the difference between $\mathrm{Ld}$ ' and Lq', which represent the differential saliency, as well as to minimize its variation with position, as this increases the burden on signal conditioning. Therefore, the issue of performance loss at high loading levels should be taken into consideration at the design stage of the machine. This work aims to achieve good overall sensorless control at high load with minimum impact on the torque producing function of the machine by the geometric dimensions of the SMPM.

A number of design studies have been carried outto optimize rotor and/or stator geometry for a specific aim, such as high torque (Wrobel and Mellor, 2004), high torque capability and low magnet volume (Vaez-Zadeh and Ghasemi, 2005; Cvetkovski et al., 2010), high torque and low THD of the back EMF (Ouyang et al., 2006), high torque and low permanent magnet (PM) weight (Bianchi and Bolognani, 1998), and minimum cogging torque (Łukaniszynet al., 2004; Abbaszadehet al., 2011; Petkovska et al., 2012). Optimized machine parameters in these studies are calculated analytically ( $\mathrm{Li}$ and Chen, 2009) or are finite element analysis (FEA) based (Wang et al., 2013; Pellegrino and Cupertino, 2010).

Numerous papers have been published looking at machine design adopting heuristic optimization techniques (Guglielmi et al., 2006; Jang et al., 2003; Jang et al., 2001; Arellano-Padilla et al., 2010; Wrobel and Mellor, 2004; Vaez-Zadeh and Ghasemi, 2005). Among these, the genetic algorithm (GA) has been successfully used as an optimization method for PM synchronous machines (Bianchi and Bolognani, 1998; Łukaniszynet al., 2004;Pellegrino and Cupertino, 2010; Wrobel and Mellor, 2008; Duan et al., 2009; Cvetkovski and Petkovska, 2008; Bianchi and Bolognani, 1997). However, as far as we are aware, this paper is the first instance of using GA optimization for the optimizing the inductance parameters for improving the sel-sensing performance in respect of position signal robustness and quality.

In our previous studies (Caner et al., 2011, 2013) same design optimisation was held using GA and sensitivities of the design variables in terms of torque and sensorless performance were discussed. But because of the optimization procedure used in these studies, the results were poor at peak loads.

In this work, GA optimization is adopted for the design of the SMPM machine to improve the self-sensing characteristics for high loading. FEA-based software was used for the calculation of these characteristics, which are arranged as design variables. A double-layer concentrated winding configuration was used in the SMPM motor.

Case studies were undertaken using three objective functions: a function that maximizes torque (density), a function that optimizes sensorless performance, and function which is a trade-off combination of the two. As a first step, the results of these studies are presented at nominal load. Then the nominal and double loading results of the sensorless performance optimization study are compared. Some of these results were published in our previous work (Caner et al., 2013). As a third step, three trade-off combination optimization results are presented for nominal, double and triple loading results and compared. It is shown that the procedure provides a wider sensorless operation region.

\section{SALIENCY CONDITIONING For SELF SENSING MACHINES}

The parameters used for representing the saliencies in PM machines are the dq incremental inductances defined as:

$L_{d}^{\prime}=\frac{\partial \psi_{d}}{\partial i_{d}} ; \quad L_{q}^{\prime}=\frac{\partial \psi_{q}}{\partial i_{q}} ; \quad L_{d q}^{\prime}=\frac{\partial \psi_{d}}{\partial i_{q}}=\frac{\partial \psi_{q}}{\partial i_{d}}$

where $\psi_{d, q}, i_{d, q}$ are standard rotating synchronous frame variable obtained from measured or derived 3-phase quantities by the appropriate transformations. In an ideal machine, the dq inductances of (1) should be independent of circumferential position $\theta$. In practice, they are not due to saturation and other space harmonics. It was shown in (Arellano-Padilla et al., 2010) that the characteristics of (1) have a strong influence on the quality of the sensorless control of the particular machine i.e. its self-sensing capability. The three quality criteria are:

$$
\begin{aligned}
& \max \left\{\Delta L\left(\theta, i_{q}\right)=\overline{L_{q}^{\prime}}\left(\theta, i_{q}\right)-\overline{L_{d}^{\prime}}\left(\theta, i_{q}\right)\right\} \\
& \min \left\{L_{q}^{\prime}\left(\theta, i_{q}\right)_{P P}\right\}, \min \left\{L_{d}^{\prime}\left(\theta, i_{q}\right)_{P P}\right\} \\
& \min \left\{\overline{L_{d q}^{\prime}}\left(\theta, i_{q}\right)\right\}
\end{aligned}
$$

where the bar denotes average inductance over $\theta=0 \rightarrow 2 \pi$ electrical. The differential inductance condition (2a) describes the requirement for saliency under all load currents. This is a serious issue since IPMSM exhibit decreasing differential saliency with load, and loss of saliency can occur within operational $i_{q}$ currents. For SMPM machines, (2a) also needs to be maximised due to the need to increase resolution and accuracy in the light of the generally small value of saliency exhibited. Condition (2b) minimizes the peak-peak ripple value of the inductance over $\theta$ (see Fig.4 for example) and represents the requirement for a sinusoidal circumferential inductance distribution for a given rotor position. This leads to a clean estimated positional signal resulting in greater bandwidths for the sensorless speed and position control loops. In practice it is found that the ripple in $L_{d}^{\prime}$ and $L_{q}^{\prime}$ change in similar proportions with machine currents and machine variables, so it is not critical which measure is taken. Condition (2c) represents the degree of dq axis coupling and for the rotor position estimator results in an estimated angle error that is a function of $i_{d, q}$. In practice, this error can be compensated by simple signal processing during real-time operation so that the minimization of (2c) is not of prime importance. This paper will consider maximizing (2a) and minimizing (2b) within the GA.

\section{ELECTRICAL MACHINE DESIGN OPTIMIZATION ENVIRONMENT}


In this study, an SMPM parametric machine model has been created in Infolytica Magnet, an FEA based software. Matlab was used to implement the optimization algorithm. The motor design geometry was created with eight variable geometric parameters which were then optimized, as will be described later, according to the different set goals. The first variable is the split ratio which indicates the ratio of the inner stator radius to the outer stator radius. The other seven design variables are shown in Fig. 1. Motor design variables and constants are summarized in Table I.

The PMs are placed on the surface of the rotor but the length of the inter-polar space can be varied. As mentioned before, the motor has 10 poles and 12 slots with doublelayer winding. Each phase consists of four coils and the windings have a concentrated structure.

The materials used for the design are shown in Table II. The outer machine dimensions were constrained for this work. The machine's geometric parameters, listed above, were optimized and, for a given convective heat transfer coefficient, the copper losses were fixed. Iron losses were ignored.

A non-linear 2D time-stepping simulation is run for each parameter change, with the current loading scaled for a fixed copper loss to determine the mean torque, on-load torque ripple and cogging torque. The quality sensorless performance parameters $\Delta L, L_{q p p}^{\prime}, L_{d p p}^{\prime}$ are calculated according to (1) and (2a) then used to calculate the fitness value which is necessary for the GA.

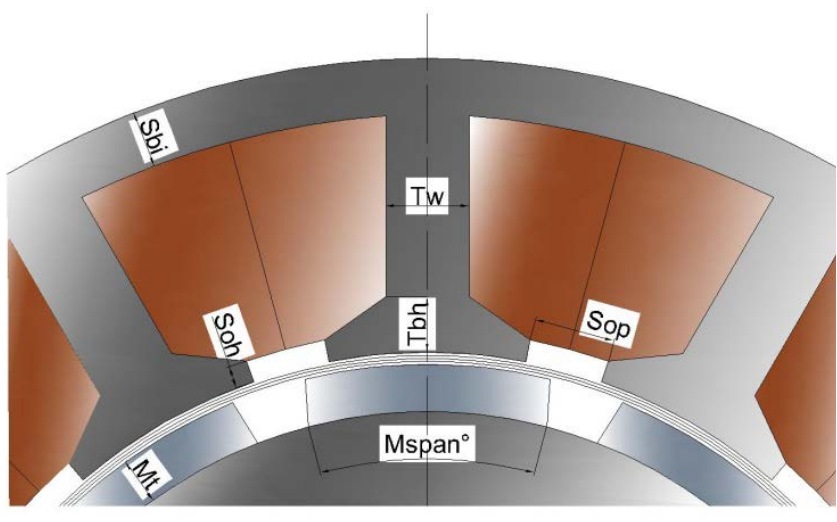

Figure 1. Design variables of motor

For better understanding of the values, the rated data for the machine have been presented in Table III.

\section{OPTIMiZation Procedure}

As a stochastic optimization method which uses a population, the GA was applied using the Matlab tool. It runs with the Matlab script which is integrated with the design software. The fundamental stages of the optimization procedure are: initialization of the GA, determining the objective function and the genetic operations.

In the matrix-formed randomly-generated initial population, one dimension is called population size the other is genes or chromosome size. The first indicates the number of individuals the other refers to the number of variables in each individual. Individuals are identified as chromosomes in GA.
The objective function, which is determined in accordance with the aim of the optimization process, allows evaluation of the individual performances using fitness values. The objective function is also called the fitness function.

Selection, crossover and mutation are segments of the GA. In order to generate a new updated population, called the child, they use fitness values beside probability.

In this study, eight variables were used as genes of the chromosome and pre-defined functions in the GA toolbox were applied to realize genetic operations. The GA parameters used for optimization are summarized in Table IV.

The flowchart given in Fig.2 demonstrates the optimization process. According to the flowchart, the GA optimization tool runs in the Matlab environment and interacts with the FEA based software to get results which are evaluated via Matlab scripts and the GA toolbox. The optimization routine stops when one of the stopping criteria reaches its limits, such as the maximum number of the generation or the number of stall generations, which is the number of generations since the last improvement of the fitness function.

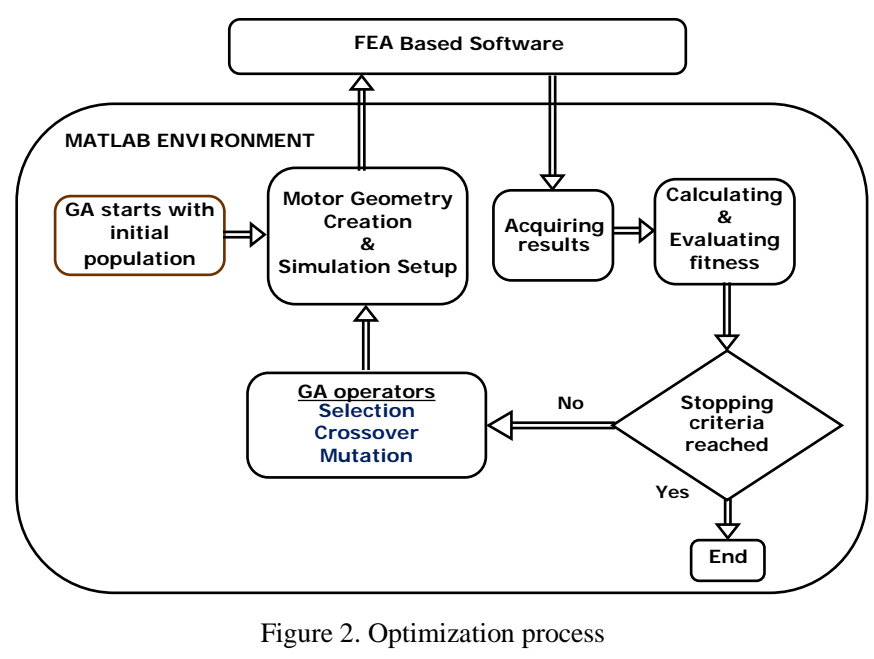

Three optimization processes are implemented using the fitness functions in $(3-5)$. The first is used to find the maximum torque with minimum torque ripple. The second is for sensorless control optimization. The last is a combination of the first two for overall optimization.

$$
f_{1}=k_{T}\left(-\frac{T_{a v g}}{30}\right)+k_{R}\left(\frac{T r}{1}\right)
$$

$f_{2}=k_{S}\left(-\left(L q_{a v g}-L d_{a v g}\right)\right)+k_{D}\left(\frac{L d p p}{0.2}\right)+k_{Q}\left(\frac{L q p p}{0.2}\right)$

$f_{3}=f_{1}+f_{2}$

In (3), $\mathrm{T}_{\mathrm{avg}}$ is the mean value of torque and $\mathrm{Tr}$ is the torque ripple and its calculation is illustrated in Fig. 3. 


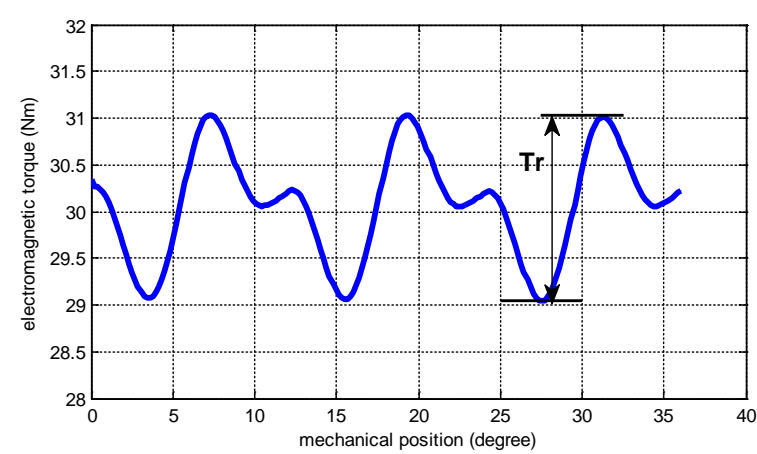

Figure 3. Calculation of torque ripple

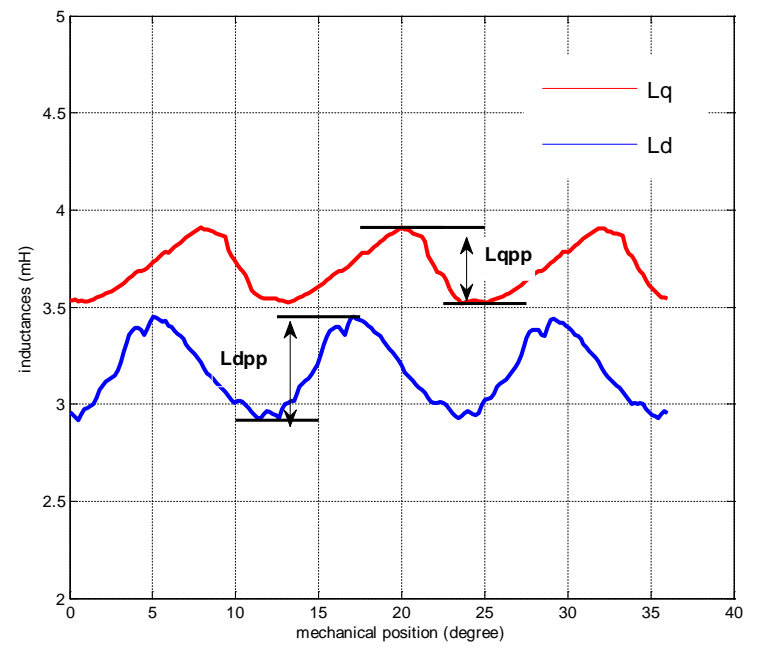

Figure 4. Calculations of peak-to-peak values of differential self inductances

In (4) Ldpp and Lqpp indicate the peak-to-peak difference in the differential self-inductance variations versus the position for the $\mathrm{d}$ and $\mathrm{q}$ axes respectively. This is illustrated in Fig. 4. These can be calculated after three simulation runs by comparing the results under nominal and two disturbed current conditions. These are also often referred to as incremental inductances. In order to calculate their values, disturbed flux variations are calculated by applying small test signals ( $\Delta \mathrm{Iq}, \Delta \mathrm{Id})$ to the $\mathrm{q}$ and $\mathrm{d}$ axis currents separately, as shown in Fig. 5. Using normal and disturbed values of flux variations, the differential selfinductance variations are calculated by using (6) and (7). 1st simulation

with normal Id, Iq

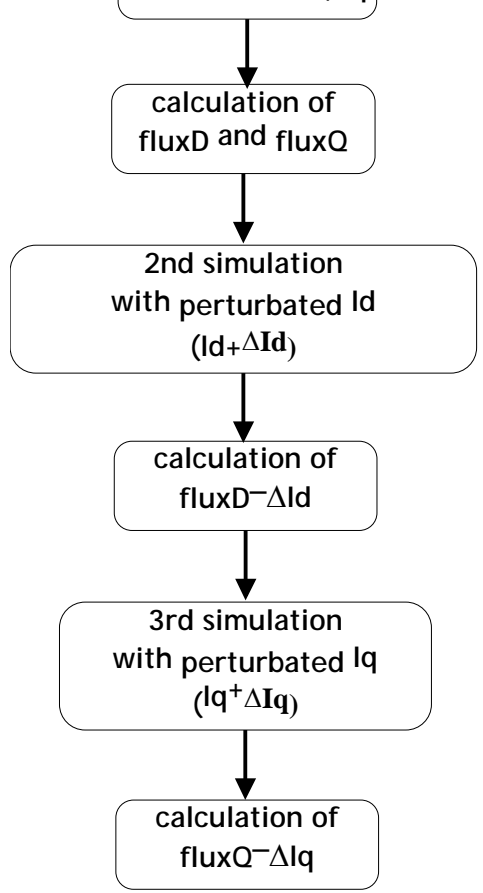

Fig. 5. Calculation of incremental inductance variations

$$
\begin{aligned}
& L_{d}^{\prime}=\frac{1}{\Delta i_{d}}\left(\text { flux } D_{\Delta i d}-\text { fluxD }\right) \\
& L_{q}^{\prime}=\frac{1}{\Delta i_{q}}\left(\text { flux }_{\Delta i q}-\text { flux } Q\right)
\end{aligned}
$$

In order not to lose any real variation of design variables used to calculate fitness, it is important to use correct simulation resolution and simulation period in the FEA software. A higher resolution provides more accurate results; however, if excessive, it will be very computationally demanding. The simulation period chosen must be as short as possible as well. In half of the electrical period, which was calculated for a $3000 \mathrm{rpm}$ speed, there are three repetitive signal variations for the torque and differential self inductances, as shown in Figs. 3 and 4. So, one-sixth of the electrical period has been chosen as a simulation period which includes one period of variations exactly. The resolution has been chosen as $0.04 \mathrm{~ms}$ so as to provide a balance between the simulation speed and the real variation error. Some of the simulation parameters are summarized in Table $\mathrm{V}$.

In this study the total simulation period for the torque optimization for Case I is 25 hours by using $0.04 \mathrm{~ms}$ as the resolution value. In order to achieve simulation results which include the incremental inductance calculation, the simulation period is approximately three times longer.

Referring to Equations (3-5), in order to maximize a part of a fitness function, the relevant part has been taken as negative (-) because the GA toolbox tries to minimize the values of these functions. In order to provide equalization, the value of each part of the fitness function is divided by certain numbers. Apart from this, weighting factors were also used to give different importance levels to each part of the equations. Here $\mathrm{k}_{\mathrm{T}}=0.9, \mathrm{k}_{\mathrm{R}}=0.1, \mathrm{k}_{\mathrm{S}}=0.6$ and $\mathrm{k}_{\mathrm{Q}}=\mathrm{k}_{\mathrm{D}}=0.2$ 
are used. Both the division and weighting values were found by the trial and error method.

Sensorless control optimization is implemented by maximizing the differential saliency and minimizing the ripple of the differential inductances. The saliency of a machine is determined by its geometry (geometric saliency) and by the levels of the magnetic saturation (saturation saliency). Both components combine to give the total saliency which can be expressed by the differential self inductances $L ' d<L$ 'q, where (') refers to the increment. This can be calculated by taking the difference of the d- and qaxis differential inductances, as seen in the first part of the fitness in (4). But in (Wrobel et al., 2011)the saliency rate has been calculated as $L ' \mathrm{q} / \mathrm{L}$ 'd.

\section{Machine Design Optimization At Nominal LoAD}

In this section, three motor designs will be described and compared with each other in terms of torque capability and sensorless performance. Although each design study was held under nominal loading, their performances were observed at high loading. Here, $\mathrm{x} 1$ current refers to nominal loading and x2 and x3 currents refer to high loading.

Each design was obtained as a result of the GA optimization algorithm using one of the fitness functions introduced in Section IV. The three fitness functions can be introduced as case studies described hereunder.

\section{Case I}

Design for both maximum continuous output torque and minimum on-load torque ripple has been carried out by using the fitness in (3). This is the typical optimization process for motors.

\section{Case II}

Sensorless performance can be determined according to the inductance profile of the machine at a given load, as mentioned in the previous section. Saliency and smoothness of the differential self inductances are the main sensorless performance indicators. In this case,the SMPM motor geometry has been designed to improve these indicators substantially using the fitness in (4) at the expense of a lower torque density.

\section{Case III}

This case includes optimization of both maximum torque with minimum torque ripple and sensorless performance. It can be called the overall optimization case which is a compromise of the two goals. This process is realized by using fitness function (5).

Three optimized motor geometries have been obtained using GA optimization under x1 current (nominal loading). SMPM motor geometry has been optimized for each goal described in each case. The resulting geometric dimensions of the three designs, with their boundaries and performance results under $\mathrm{x} 1, \mathrm{x} 2$ and $\mathrm{x} 3$ currents, are summarized in Table VI (Caner et al., 2013).

The limits of the design variables have been chosen by undertaking pre-optimization studies in order to maximize the torque capability of the motor. Naturally, the more magnetic material used, the more the torque capability increases; however, magnet dimensions must be limited due to cost. Therefore, a $150^{\circ}$ span and a $4 \mathrm{~mm}$ thickness have been accepted as maximum values of magnet span and thickness, respectively.

The cylindrical outer surface is assumed as an area from which all the losses are transferred as heat.

According to the results above, the performance indicator values were improved in terms of each case goal at nominal load. As expected, the relative performance in terms of torque producing capability is maintained, with Case I producing the maximum torque throughout the loading range. Sensorless performance deteriorates drastically for all the three cases in the $\mathrm{x} 2$ current condition and is almost fully lost in the $\mathrm{x} 3$ current condition for all cases. As expected, sensorless performance is at a maximum for Case II in the $\mathrm{x} 1$ and $\mathrm{x} 2$ currents but not in $\mathrm{x} 3$.

In order to visually evaluate the differences among the three machine geometries, they are illustrated for each case in Fig. 6. The first notable thing is that the slots are wider and the stator back iron is thinner in the designs shown in Case II and III. This pushes the stator core operating point to a higher flux density at the simulated load point.

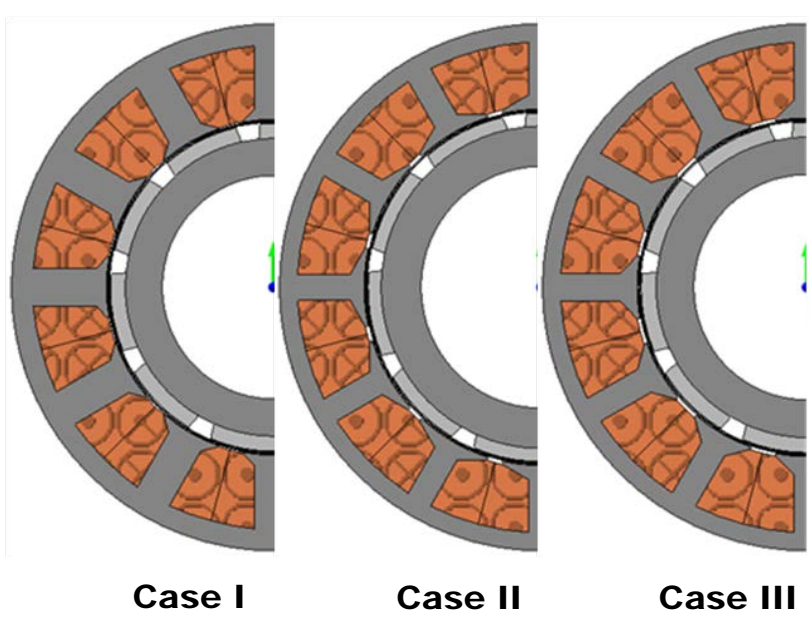

Figure 6. Half part of the optimized geometry of motor a) in Case I b) in Case II and c) in Case III

\section{Machine Design Optimization At High LoAd}

This section will look at improving the sensorless capability of the machine at high load. Section V demonstrated that the machine lost its self-sensing capability when high currents were applied. As discussed in the introduction, an important aspect of the sensorless control of these machines is their performance at high current loading. Within this operating region, the machine's armature reaction tends to saturate the stator core with the consequence of losing the differential saliency and consequently control.

Here, in Case II, motor design optimization has been repeated using $\mathrm{x} 3$ current condition. Thus, differences between the optimized geometries in terms of sensorless design under the nominal and high loading conditions can be observed as in Fig. 7. Although the shapes of the slots area of the motor geometry are similar to the nominal loading condition, the thickness of the stator parts, like the back iron, and tooth width are greater. This allows for a lower magnetic saturation operating point. 


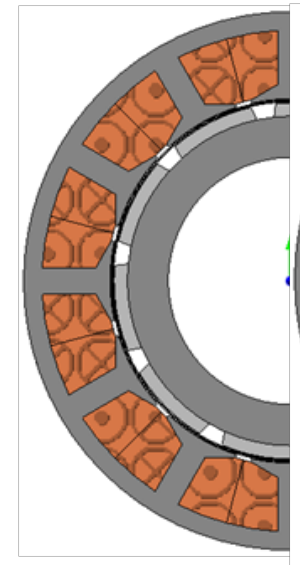

Case II at nominal load

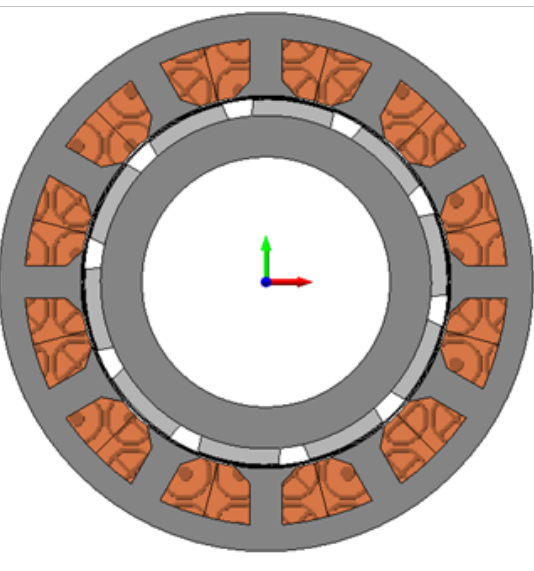

Case II at high load
Figure 7. Optimized geometry of motor in Case II at nominal and high load

In addition to the design optimization above, the same motor geometry with the same design constants and variables will be optimized using two different methods and their performances will be discussed in terms of sensorless control capability. The first used $\mathrm{x} 2$ and $\mathrm{x} 3$ currents separately for Case III, which is overall optimization. The second is the proposed technique which includes both $\mathrm{x} 1$ and $\mathrm{x} 2$ current simulations. The latter is a combination of torque optimization and a sensorless optimization. It calculates the torque part of the fitness using $\mathrm{x} 1$ current and the sensorless part using $\mathrm{x} 2$ current. The differences in the two design procedures are as shown in the flowchart in Fig. 8. Here, the nominal loading results of the overall optimization were added as Design A, which has already been presented in a previous chapter as Case III for comparison.

Performance indicator results of the optimized machine designs for each loading condition (x1, x2 and $\mathrm{x} 3$ rated currents) are given in Table VII. Here, Designs B and C indicate the optimization for Case III under $\mathrm{x} 2$ and $\mathrm{x} 3$, respectively. Design procedure in Designs $\mathrm{A}, \mathrm{B}$ and $\mathrm{C}$ is same as in (Caner et al., 2011, 2013).

Design $\mathrm{D}$ indicates the results of the proposed technique and Design $\mathrm{A}$ is the $\mathrm{x} 1$ current result of the overall optimization and has been added for comparison.

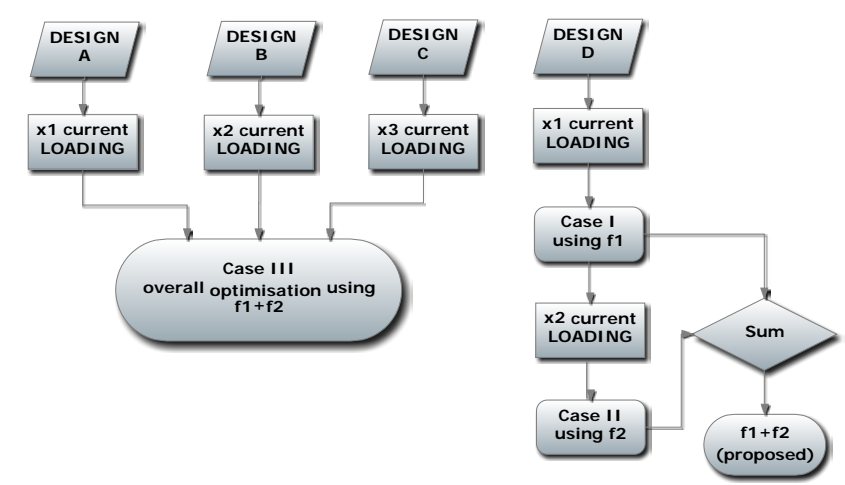

Figure 8. Difference of calculation objective functions between Designs A, $\mathrm{B}, \mathrm{C}$ and proposed D.

In nominal loading, Designs $\mathrm{A}$ and $\mathrm{B}$ are better in terms of torque results but Designs $\mathrm{A}$ and $\mathrm{D}$ are successful in terms of sensorless performance. Design $C$ is the worst for both.

In the $\mathrm{x} 2$ rated current condition, Design $\mathrm{B}$ is the best, Designs $A$ and D are acceptable and Design $C$ is the worst in terms of torque performance. Design $\mathrm{D}$ is superior in terms of sensorless performance. In Design A, however, although the saliency is good, the $L_{d}$ ripple is very high.

In the $\mathrm{x} 3$ rated current condition, Designs B and D are acceptable and Design $C$ is the worst in terms of torque performance. Design $\mathrm{D}$ is superior in terms of sensorless performance. Although the saliency is slightly higher from Design C, Design C actually has negative saliency for both the $\mathrm{x} 2$ and $\mathrm{x} 3$ rated currents. However, the $\mathrm{L}_{\mathrm{d}}$ ripple is a problem in Design D.

The overall optimization used in Designs A, B and C was held under only one loading condition. The proposed technique enables a trade-off between nominal and high loads in addition to overall optimization, which has a tradeoff already in the nature between torque and sensorless performance.

The geometric results of the variables for Designs B, C and $\mathrm{D}$, with the boundaries used during optimization, are given in Table VIII.

Incremental inductance variations with position angle visualize the sensorless performances. The $d$ and $q$ inductance variations of Designs $\mathrm{B}$ and $\mathrm{C}$ are given in Figs. 9 and 10 for $\mathrm{x} 2$ and $\mathrm{x} 3$ loadings, respectively. It can be said that these designs present good sensorless results for the loadings which are optimized. However, it must be noted that Design C has negative saliency here. Despite this, the proposed sensorless design is good for both light and heavy loads (Figs. 11-13). The ripple problem can be seen in Fig. 13.

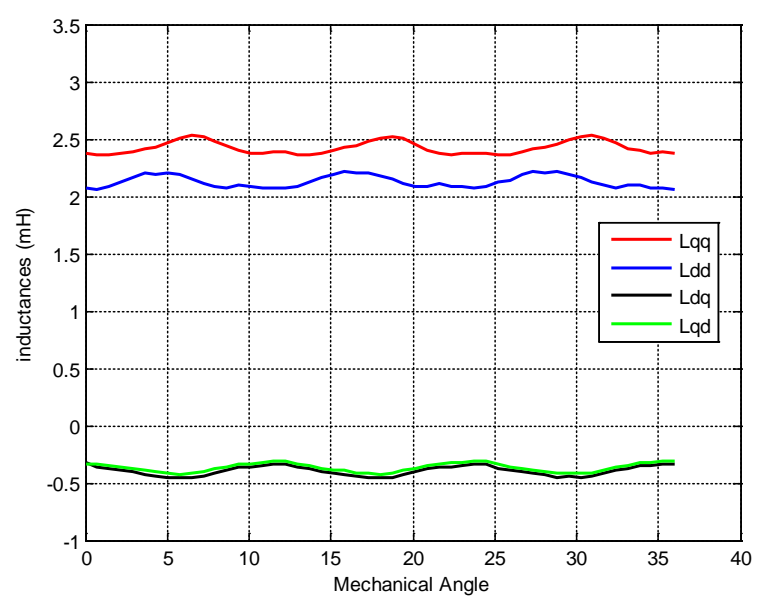

Figure 9. Design B at x2 loading 


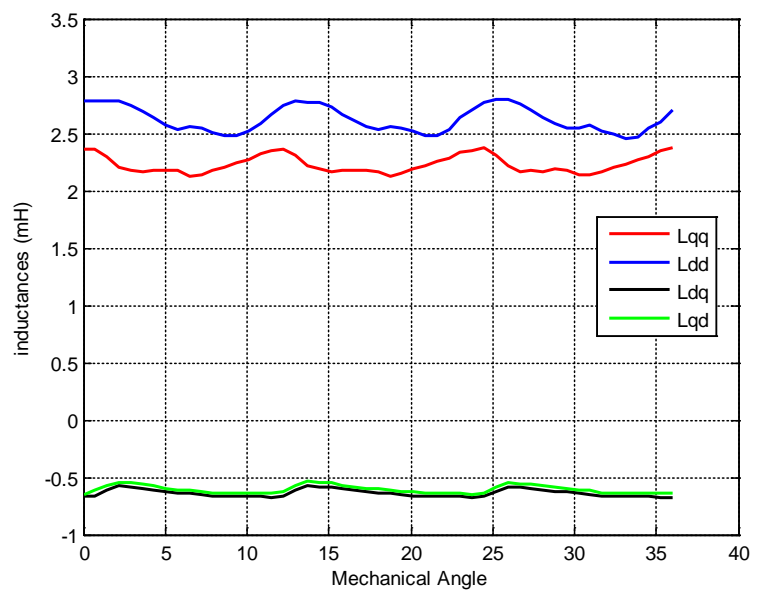

Figure 10. Design C at x3 loading presents negative saliency

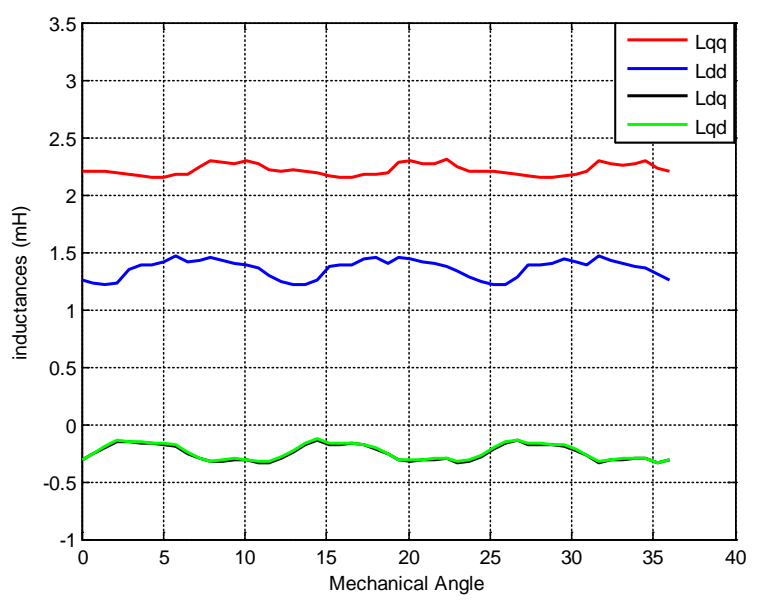

Figure 11. Design D at x1 loading

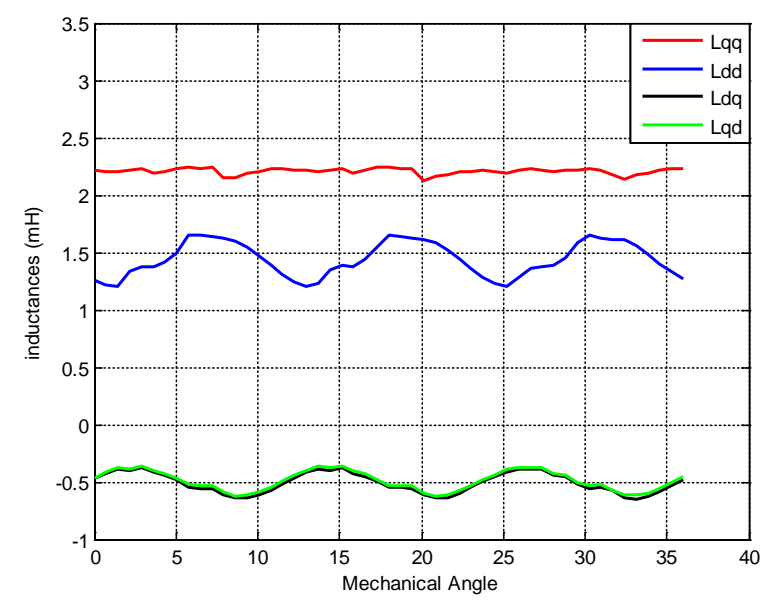

Figure 12. Design D at x2 loading

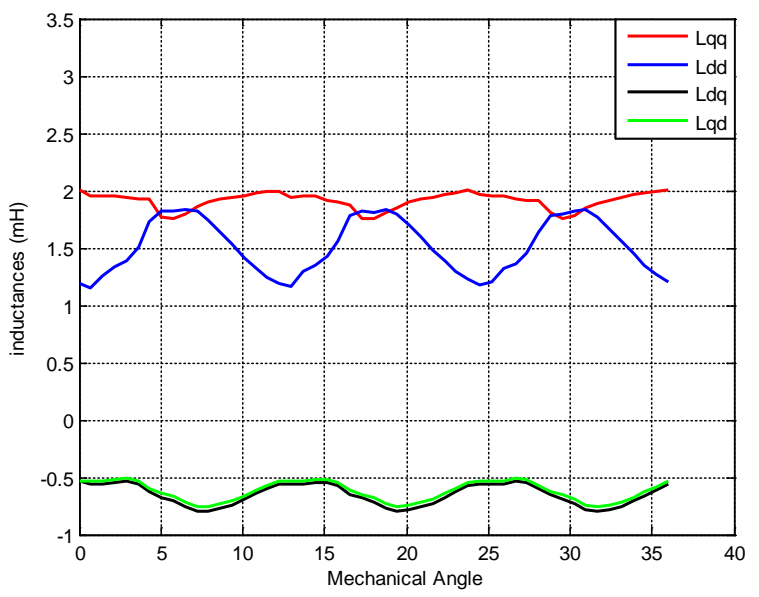

Figure 13. Design D at x3 loading

The results of saliency versus a 25\% load-step increase for each design have been shown in Fig. 14. Design D can be accepted as the best design for sensorless performance in a wide loading range. It is apparent that, by using proper optimization procedures, good sensorless performance of the motor can be achieved at both high and nominal loadings. The more the loading is increased, the more it is difficult to optimize the machine in terms of sensorless control without affecting the overall performance at nominal load.

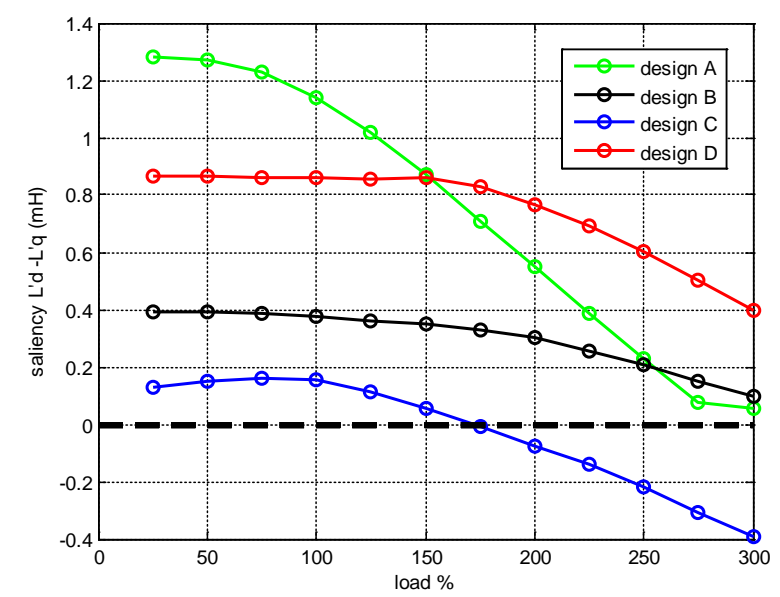

Figure 14.Saliency vs loading

Trade-off concept can be expressed by quantifying sensorless and torque performance. Equation (8) can be used to find the trade-off value by using maximum values of torque and saliency for each loading. Here trade off is the multiplication of the torque and saliency rates. Tmax and Smax values were taken as the biggest value in Table VI and VII for each loading.

Trade off $=\left(1-\frac{T \text { max }-T}{T \text { max }}\right)\left(1-\frac{S \text { max }-S}{S \max }\right)$

The trade-off values have been calculated for each loading and shown in Fig. 15. According to this Design D can be accepted as the best at high loading. 


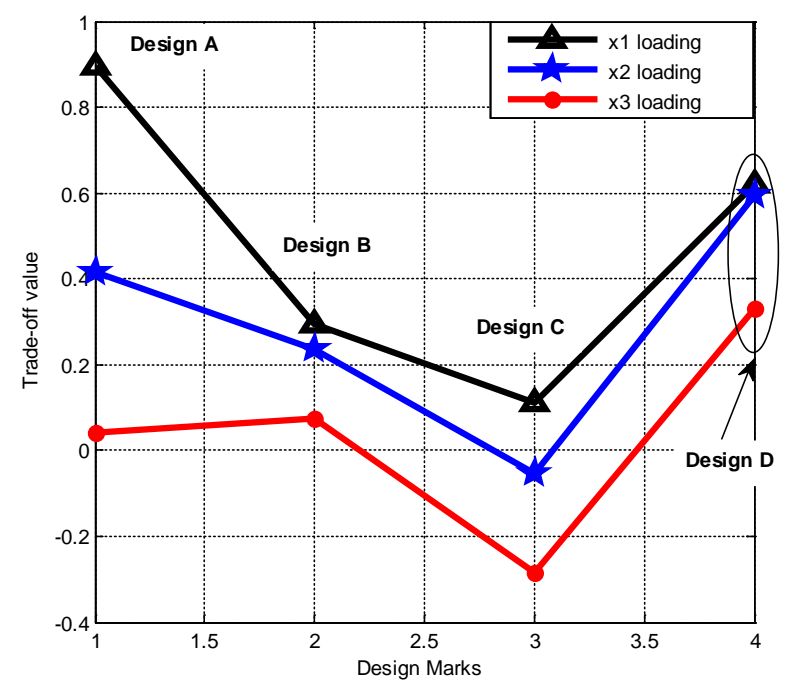

Figure 15.Trade-off values each design between Torque and Saliency

\section{CONCLUSIONS}

This paper demonstrates that the self-sensing properties of a SMPM machine can be improved for both high and nominal loads using proper optimization strategies at the expense of $10-15 \%$ more torque capability. A new combined strategy was implemented using a GA to optimize both the torque and sensorless properties of the machine.

Optimization is not trapped by local minima because of the stochastic nature of the GA, which generates a parameter space for the geometric data of the topology of both the stator and rotor.

Being considered to be the sensorless features at the design stage of the machine can provide reliable operation at high loads as well.

It was seen that, although optimization at each loading itself is successful only for the loading at hand, the proposed optimization shows superior sensorless performance for all loadings, except for the ripple problem in $L_{d}{ }_{d}$ at the $x 3$ current condition.

It was also seen that there was a trade-off between torque capability and sensorless performance. Therefore, GA optimization can be applied looking pareto front as a future work.

\section{ACKNOWLEDGMENT}

We would like to thank the Scientific and Technical Research Council of Turkey (TUBITAK) for their support. 